\title{
Can the Stone Scoring Systems Be Used to Predict Infective Complications of Retrograde Intrarenal Surgery?
}

\author{
Samet Senel Cuneyt Ozden Yilmaz Aslan Yalcin Kizilkan \\ Cevdet Serkan Gokkaya Binhan Kagan Aktas \\ Department of Urology, Ankara City Hospital, Ankara, Turkey
}

\section{Highlights of the Study}

- Independent risk factors for the development of infective complications after retrograde intrarenal surgery (RIRS) are age $>50$ years, surgical duration $>60 \mathrm{~min}$, and a high R.I.R.S. score.

- The R.I.R.S. score can be used to predict infective complications after RIRS.

- Resorlu-Unsal Stone Score and modified Seoul National University Renal Stone Complexity Score are not useful for predicting infective complications after RIRS.

\section{Keywords}

Infective complication · Nephrolithiasis · Retrograde

intrarenal surgery $\cdot$ Stone scoring systems

\section{Abstract \\ Objective: We aimed to evaluate whether the retrograde in- trarenal surgery (RIRS) scoring systems (Resorlu-Unsal Stone Score [RUSS], modified Seoul National University Renal Stone Complexity Score [modified S-ReCS], and R.I.R.S. score) can predict the infective complications after RIRS. Subject and Methods: A total of 581 patients who underwent RIRS for kid- ney stones were included in the study. All patients were eval- uated for demographic data, medical history, radiological im- aging methods before surgery, duration of surgery, and hos- pitalization time after surgery. Stone laterality, stone burden, stone size, stone density, the number of stones, stone local- ization, the presence of congenital kidney anomaly, and soli- tary kidney were evaluated preoperatively by computed to- mography. The RUSS and modified S-ReCS and R.I.R.S. score of all patients were determined. Results: Infective complica-}

Karger@karger.com www.karger.com/mpp

Karger $\stackrel{\text { ' }}{5}$
(C) 2022 The Author(s)

Published by S. Karger AG, Basel

This is an Open Access article licensed under the Creative Commons Attribution-NonCommercial-4.0 International License (CC BY-NC) (http://www.karger.com/Services/OpenAccessLicense), applicable to the online version of the article only. Usage and distribution for commercial purposes requires written permission. tions were detected in $47(8.1 \%)$ patients who underwent RIRS. Fever developed in 27 subjects (4.6\%), urinary infection in $15(2.5 \%)$, sepsis in $2(0.3 \%)$, and septic shock in $3(0.5 \%)$ patients. In multivariate logistic regression analysis, age (OR $=1.8 ; 95 \% \mathrm{Cl}=1-3.4 ; p=0.049$ ), surgical duration of $>60 \mathrm{~min}$ $(\mathrm{OR}=1.9 ; 95 \% \mathrm{Cl}=1.1-3.5 ; p=0.027)$, and high R.I.R.S score $(\mathrm{OR}=8.9 ; 95 \% \mathrm{Cl}=1.9-42.4 ; p=0.006)$ were shown to be independent risk factors for the infective complications after RIRS. A receiver operating characteristic curve analysis showed that the R.I.R.S. score can be used as a marker to predict infective complications ( $\mathrm{UUC}=0.619, \mathrm{Cl}=0.55-0.69 ; p=$ 0.007). Conclusion: We suggest that the R.I.R.S. score can be used to predict infective complications in RIRS.

(c) 2022 The Author(s).
Published by S. Karger AG, Basel

\section{Introduction}

Conservative, medical, and surgical treatments are among the treatment methods for stones in the urinary system. The primary goal in the treatment of kidney 
stones is to provide a maximally stone-free rate (SFR) with minimal morbidity. The treatment of kidney stones has changed significantly in the last 30 years. Treatment options now include percutaneous nephrolithotomy (PNL), extracorporeal shock wave lithotripsy (SWL), and retrograde intrarenal surgery (RIRS), which are less invasive than open surgery [1].

In recent years, the use of RIRS in the treatment of upper urinary tract stones is on the rise worldwide. One of the most important reasons for the frequent use of RIRS is that it has a lower complication rate than PNL and a higher SFR than SWL. RIRS continues to be increasingly used as there is an increase in the number of its indications and as surgeons are gaining more experience in performing it [2].

Complications associated with RIRS include urinary infection, bleeding, subcapsular hematoma, ureteral injury, avulsion, and ureteral stricture. Infective complications are the most common among these and can even become fatal in some cases [3]. It has been reported that stone scoring systems that predict the SFR after PNL can also predict postoperative complications [4]. However, to the best of our knowledge, there are no published reports evaluating the relationship between the RIRS scoring systems and infective complications after RIRS. This study aimed to ascertain whether the Resorlu-Unsal Stone Score (RUSS), modified Seoul National University Renal Stone Complexity Score (S-ReCS), and R.I.R.S. score can predict the infective complications after RIRS [5-7].

\section{Subjects and Methods}

\section{Patients and Clinical Data}

This study was prepared in accordance with the principles of the Declaration of Helsinki and was approved by the local Ethics Committee (Approval No. E1-20-451). Informed consent was obtained from each subject. Totally, 686 RIRS procedures performed in our clinic between January 2013 and May 2020 were analyzed retrospectively from the hospital information database. Cases without adequate data and secondary cases were excluded from the study. A total of 581 patients who underwent primary RIRS for kidney stones were included in the study. Pre-, intra-, and postoperative data of all patients included in the study were obtained from the hospital information database. All patients were evaluated for demographic data, medical history, and radiological imaging methods before surgery as well as the duration of surgery and hospitalization time after surgery.

Stone laterality (right-left), stone burden $\left(\mathrm{mm}^{2}\right)$, stone size $(\mathrm{mm})$, stone density (Houndsfield unit [HU]), the number of stones (single and multiple), stone localization (pelvis, upper calyx, middle calyx, lower calyx, and multiple localization), the presence of congenital kidney anomaly, solitary kidney, and preoperative stenting were evaluated preoperatively by computed tomography.
Stone burden was defined as the two-dimensional area determined by multiplying the longest diameter by the perpendicular diameter of the stone. In cases with multiple stones, stone size was defined as the sum of the burdens of each stone.

All patients were scored according to stone scoring systems. In RUSS, 4 parameters were evaluated. These parameters included stone size $>20 \mathrm{~mm}$, the infundibulopelvic angle (IPA) $<45^{\circ}$ for lower pole stones, stone number in different calyces, and abnormal kidney anatomy such as horseshoe kidney or pelvic kidney. 1 point was given for each parameter, and patients were scored with a score between 0 and 4 points [5]. The modified S-ReCS was scored according to the localization of the stone in the kidney. These localizations were the renal pelvis, upper and lower major calyx groups, anterior and posterior upper minor calyx groups, and middle and inferior calyces. 1 point was given for each location and 1 more point was added if the stone, or one of the stones, was located in the lower calyx. In this way, the total modified S-ReCS was calculated to be between 1 and 12 points [6]. In the R.I.R.S. score, the parameters of stone density (HU), IPA, infundibular length, stone size $(\mathrm{mm})$, and the presence of lower pole stone were checked. 1 point was given if the stone density was $\leq 1,000 \mathrm{HU}$, and 2 points were given if stone density was $>1,000 \mathrm{HU}$. The IPA was calculated for lower pole stones. 1 point was given if the stone was not in the lower pole. 2 points were given if the stone was in the lower pole and the IPA was $>25^{\circ}$, and 3 points were given if the stone was in the lower pole and the IPA was $\leq 30^{\circ}$ [7].

As suggested in the original studies [5-7], the patients were divided into 2 groups according to RUSS ( 0 points and 1-4 points), and 3 groups according to the modified S-ReCS (low score: 1-2 points, medium score: $3-4$ points, high score $5-12$ points) [6], and 4 groups according to R.I.R.S. score (low score: $4-5$ points, medium score: $6-8$ points, high score: $9-10$ points). To calculate these scores, infundibular length was measured as the distance from the furthest point under the calyx containing the stone to the midpoint of the lower edge of the renal pelvis. IPA was measured as the internal angle formed by the intersection of the ureteropelvic axis and the central axis of the infundibulum of the lower pole.

\section{Surgical Technique}

Preoperative urine cultures of all patients were sterilized. Prophylaxis with $2 \mathrm{~g}$ of cefazolin was administered intravenously to all patients within $1 \mathrm{~h}$ before surgery. RIRS was performed in all patients under general anesthesia in the lithotomy position. Ureterorenoscopy was performed with a $9.5 \mathrm{~F}$ rigid ureterorenoscope (KARL STORZ, Tuttlingen, Germany) before RIRS. In cases where ureteral dilatation was sufficient, the access sheath was used and after the access sheath reached the collecting system, the collecting system was reached by entering through the access channel with a 7.5 F flexible ureterorenoscope (KARL STORZ, Flex X2, GmbH, Tuttlingen, Germany). In cases where the access sheath could not be delivered to the collecting system due to insufficient ureteral dilatation, a DJ stent was placed and RIRS was postponed for 2 weeks. After 2 weeks, the access sheath was used in these cases, too. The stone was fragmented using a holmium-yttrium-aluminumgarnet laser $(200-365 \mu \mathrm{m})$ sent from the working channel of the flexible ureterorenoscope. The DJ stent and urethral catheter were used in all cases at the end of the operation. The patients were evaluated with computed tomography in the postoperative first month. Success was defined as the absence of stones in the urinary system or the presence of asymptomatic residual stones $\leq 2 \mathrm{~mm}$ in size. 
Table 1. Preoperative clinical and demographic data of patients

\begin{tabular}{|c|c|c|}
\hline & Mean \pm SD & $N(\%)$ \\
\hline \multicolumn{3}{|l|}{ Age, years } \\
\hline$\leq 50$ & \multirow{2}{*}{$46.1 \pm 14.2$} & $3540.9)$ \\
\hline$>50$ & & $227(39.1)$ \\
\hline \multicolumn{3}{|l|}{ Gender } \\
\hline Male & & $366(63)$ \\
\hline Female & & $215(37)$ \\
\hline \multicolumn{3}{|l|}{ Stone laterality } \\
\hline Right & & $276(47.5)$ \\
\hline Left & & $305(52.5)$ \\
\hline \multicolumn{3}{|l|}{ Stone burden, $\mathrm{mm}^{2}$} \\
\hline$<100$ & \multirow{3}{*}{$169.5 \pm 141$} & $184(31.7)$ \\
\hline $100-200$ & & $242(41.7)$ \\
\hline$>200$ & & $155(26.6)$ \\
\hline \multicolumn{3}{|l|}{ Stone density, HU } \\
\hline$\leq 1,000$ & \multirow{2}{*}{$962.1 \pm 327.2$} & $305(52.5)$ \\
\hline$>1,000$ & & $276(47.5)$ \\
\hline \multicolumn{3}{|l|}{ Stones, $n$} \\
\hline Single & & $376(64.7)$ \\
\hline Multiple & & $205(35.3)$ \\
\hline \multicolumn{3}{|l|}{ Stone localization } \\
\hline Pelvis & & $239(41.1)$ \\
\hline Upper calyx & & $36(6.2)$ \\
\hline Middle calyx & & $51(8.8)$ \\
\hline Lower calyx & & $168(28.9)$ \\
\hline Multiple localization & & $87(15)$ \\
\hline History of urinary tract infection & & $25(6.8)$ \\
\hline History of stone surgery & & $224(38.6)$ \\
\hline History of SWL & & $46(7.9)$ \\
\hline Presence of congenital renal anomaly & & $14(2.4)$ \\
\hline Presence of solitary kidney & & $21(3.6)$ \\
\hline Presence of preoperative stent & & $51(8.8)$ \\
\hline
\end{tabular}

SD, standard deviation; HU, Hounsfield unit; SWL, shock wave lithotripsy.

Infective complications after RIRS were defined as fever (body temperature $>38^{\circ} \mathrm{C}$ continuing for $48 \mathrm{~h}$ during hospital stay), urinary infection (urinary and/or blood culture positivity), sepsis (urinary infection and the presence of $\geq 2$ Sequential Sepsis-related Organ Failure Assessment [SOFA] score), and septic shock (sepsis accompanied by serum lactate level $>2 \mathrm{mmol} / \mathrm{L}$ and vasopressor need for mean arterial pressure $>65 \mathrm{~mm} \mathrm{Hg}$ ) [8].

\section{Statistical Analysis}

The coding and statistical analysis of the data were done on the computer, using the SPSS 22 software (IBM SPSS Statistics, IBM Corporation, Chicago, IL, USA) package. Descriptive statistical data for continuous variables were expressed in terms of the mean and median, whereas categorical variables were expressed in terms of frequency and percentages. Receiver operating characteristic curves (ROCs) were created to evaluate the predictive role of the modified S-ReCS, RUSS, and R.I.R.S. score in terms of postoperative infective complication rates. Univariate and multivariate logistic regression models were used to identify risk factors for postoperative infective complications. The statistical significance level was accepted as 0.05 in all analyses.

R.I.R.S. Score and Infective Complications after RIRS
Table 2. Perioperative and postoperative data of patients

\begin{tabular}{lll}
\hline & Mean \pm SD & $N(\%)$ \\
\hline $\begin{array}{l}\text { Surgical duration, min } \\
\quad<60\end{array}$ & $54.3 \pm 18.3$ & \\
$\quad$ & & $352(60.6)$ \\
Hospitalization, days & $229(39.4)$ \\
Postoperative stent duration, days & $1.8 \pm 3.9$ & \\
Success rate & $19.2 \pm 8.4$ & \\
Intraoperative complications & & $426(73.3)$ \\
$\quad$ No & \\
$\quad$ Hematuria & $457(78.7)$ \\
$\quad$ Minimal mucosal injury & $87(14.9)$ \\
$\quad$ Ureteral perforation & $29(4.9)$ \\
Retroperitoneal abscess & $5(0.9)$ \\
Perirenal hematoma & $2(0.4)$ \\
Infective complications & $1(0.2)$ \\
$\quad$ No & & \\
Fever & & $534(91.9)$ \\
Urinary tract infection & $27(4.7)$ \\
Sepsis & $15(2.6)$ \\
Septic shock & $2(0.3)$ \\
\hline SD, standard deviation. & $3(0.5)$ \\
\hline
\end{tabular}

\section{Results}

The mean age of the 581 patients who underwent RIRS was included in this study. All operations were performed by 3 different surgeons with at least 10 years of experience in RIRS. Preoperative clinical and demographic data of patients are shown in Table 1.

The success rate of the RIRS was $73.3 \%$. The mean surgical duration was $54.3 \pm 18.3 \mathrm{~min}$ and the patients were hospitalized for a mean period of $1.8 \pm 3.9$ days postoperatively. A total of 123 (21.2\%) patients had intraoperative complications. Infective complications were detected in $47(8.1 \%)$ patients (Table 2). A total of $27(4.6 \%)$ had only fever, $15(2.5 \%)$ had urinary infection, $2(0.3 \%)$ had sepsis, and 3 (0.5\%) had septic shock. Escherichia coli (8 patients), Pseudomonas aeruginosa (5 patients), Enterococcus faecalis (4 patients), Candida glabrata (1 patient), Candida dubliniensis (1 patient), and Klebsiella pneumoniae (1 patient) growths were detected in the urine cultures of the patients who had urinary infection, sepsis, and septic shock.

According to the multivariate logistic regression analysis, the independent risk factors for the development of infective complications after RIRS were age $>50$ years $(\mathrm{OR}=1.8 ; 95 \% \mathrm{CI}=1-3.4 ; p=0.049)$, surgical duration of $>60 \min (\mathrm{OR}=1.9 ; 95 \% \mathrm{Cl}=1.1-3.5 ; p=0.027)$, and a high R.I.R.S score $(\mathrm{OR}=8.9 ; 95 \% \mathrm{CI}=1.9-42.4 ; p=0.006)$ (Table 3). 
Table 3. Univariate and multivariate logistic regression analysis of factors associated with infective complications

\begin{tabular}{|c|c|c|c|c|}
\hline & \multicolumn{2}{|l|}{ Univariate } & \multicolumn{2}{|l|}{ Multivariate } \\
\hline & OR $(95 \% \mathrm{Cl})$ & $p$ value & OR $(95 \% \mathrm{Cl})$ & $p$ value \\
\hline Gender (female) & $1.2(0.6-2.2)$ & 0.613 & & \\
\hline Age ( $>50$ years) & $1.9(1-3.4)$ & 0.041 & $1.8(1-3.4)$ & 0.049 \\
\hline \multicolumn{5}{|l|}{ Stone burden, $\mathrm{mm}^{2}$} \\
\hline$<100$ & 1 & & & \\
\hline $100-200$ & $1.5(0.7-3.3)$ & 0.329 & & \\
\hline$>200$ & $2.3(0.9-3.8)$ & 0.216 & & \\
\hline Stone density (>1,000 HU) & $1.7(0.9-3.1)$ & 0.087 & & \\
\hline \multicolumn{5}{|l|}{ Localization } \\
\hline Pelvis & 1 & & & \\
\hline Upper calyx & $1.3(0.3-4.6)$ & 0.718 & & \\
\hline Middle calyx & $2.2(0.9-5.7)$ & 0.099 & & \\
\hline Lower calyx & $1.4(0.7-2.8)$ & 0.404 & & \\
\hline Multiple & $1.1(0.4-2.7)$ & 0.949 & & \\
\hline Laterality (right) & $1.1(0.6-1.9)$ & 0.838 & & \\
\hline History of urinary tract infection & $1.8(0.7-4.5)$ & 0.205 & & \\
\hline History of stone surgery & $1.4(0.8-2.6)$ & 0.227 & & \\
\hline History of SWL & $1.2(0.6-2.5)$ & 0.645 & & \\
\hline Presence of congenital kidney anomaly & $0.9(0.1-6.8)$ & 0.895 & & \\
\hline Presence of solitary kidney & $2.8(0.9-8.8)$ & 0.072 & & \\
\hline Presence of preoperative stent & $0.9(0.4-2.2)$ & 0.914 & & \\
\hline Presence of intraoperative complication & $1.1(0.6-2.1)$ & 0.849 & & \\
\hline Surgical duration ( $\geq 60 \mathrm{~min}$ ) & $2.2(1.1-4.1)$ & 0.022 & $1.9(1.1-4.2)$ & 0.027 \\
\hline \multicolumn{5}{|l|}{ RUSS } \\
\hline 0 & 1 & & & \\
\hline 1 & $1.2(0.6-2.5)$ & 0.538 & & \\
\hline 2 & $0.9(0.3-2.9)$ & 0.975 & & \\
\hline$\geq 3$ & $1.1(0.2-4.9)$ & 0.906 & & \\
\hline \multicolumn{5}{|l|}{ Modified S-ReCS } \\
\hline $1-2$ & 1 & & & \\
\hline $3-4$ & $1.3(0.7-2.7)$ & 0.408 & & \\
\hline $5-12$ & $1.1(0.3-3.6)$ & 0.945 & & \\
\hline \multicolumn{5}{|l|}{ R.I.R.S scoring system } \\
\hline $4-5$ & 1 & & 1 & \\
\hline $6-8$ & $4.4(1.7-11.4)$ & 0.002 & $4.7(1.8-12.1)$ & 0.002 \\
\hline $9-10$ & $6.6(1.5-30)$ & 0.015 & $8.9(1.9-42.4)$ & 0.006 \\
\hline
\end{tabular}

HU, Hounsfield unit; SWL, shock wave lithotripsy; RUSS, Resorlu-Unsal Stone Score; S-ReCS, Seoul National University Renal Stone Complexity Score.

ROC curves were used to evaluate the effectiveness of the scoring systems in predicting infective complications after RIRS (Fig. 1). The ROC curve analysis showed that the R.I.R.S. score can be used as a marker to predict infective complications (AUC $=0.619, \mathrm{CI}=0.55-0.688$; $p=$ 0.007). According to Youden's index, the cutoff value of R.I.R.S. score for infective complications after RIRS was 5.5 point (sensitivity 0.894 , specificity 0.554 ). RUSS and modified S-ReCS were far from predicting infective complications (respectively, AUC $=0.515, \mathrm{CI}=0.429-0.601$; $p=0.73$ and $\mathrm{AUC}=0.542, \mathrm{CI}=0.457-0.626 ; p=0.345$ ).

\section{Discussion}

We aimed to evaluate the ability of scoring systems to predict infective complications that may develop after RIRS. We concluded that RUSS and modified S-ReCS scoring systems are insufficient in predicting infective complications. In our study, the R.I.R.S. score has been shown to be an independent factor in predicting postoperative infective complications. To our knowledge, this is the first study to confirm that a scoring system can be used to predict infective complications after RIRS. 


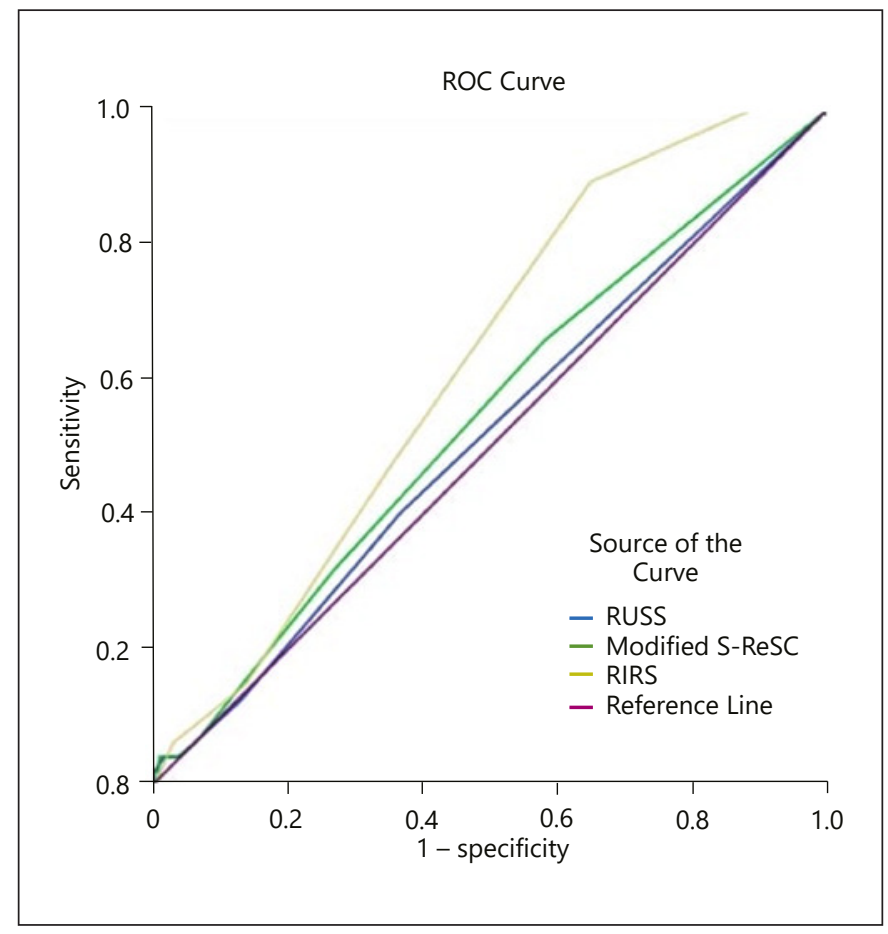

Fig. 1.ROC curve comparing the efficacy of scoring systems in predicting infective complications after retrograde intrarenal surgery.

With the development of minimally invasive surgical techniques, scoring systems have been developed to evaluate the postoperative success and complication rates of the surgical methods used in the management of kidney stones $[5,9]$. The Guy's stone score, Clinical Research Office of Endourological Society nomogram, S.T.O.N.E score, and S-ReCS have been used to predict an SFR and complications after PNL [10-12]. Parameters that affect the prediction of success rate and treatment selection have been identified and are still in use for SWL, a surgical method that has been used for a long time and continues to be used even today [13].

Although RIRS is accepted as a safe surgical procedure, postoperative infective complications are the most common complications reported with a rate of $5.7 \%-18.3 \%$ and can have fatal consequences $[8,14,15]$. However, it should be noted that systemic inflammatory response syndrome (SIRS) criteria were used for the definition of sepsis and septic shock in these studies. However, at the Third International Consensus Meeting held in 2016 (Sepsis 3), it was stated that the presence of organ dysfunction was an important parameter in the differentiation of sepsis from uncomplicated infection and the use of the total SOFA score instead of the SIRS criteria in this differentiation was recommended [16]. In our study, in which the definition of sepsis was made according to the new criteria, the postoperative infective complication rate was found to be $8.1 \%$, whereas $4.6 \%$ of the patients had only fever, $2.6 \%$ had urinary system infection, $0.3 \%$ had sepsis, and $0.5 \%$ had septic shock. To the best of our knowledge, this study is the first of its kind in which infective complications after RIRS were defined according to the total SOFA score; we believe that the lower rate of patients with sepsis compared with that in other studies is related to the use of this new classification.

According to the results of a recent review, infective complications after RIRS are more common in patients with a high Charlson Comorbidity Index, advanced age, female gender, long-term preoperative ureteral catheter, neurogenic bladder, and high body mass index [17]. The stone burden was observed to be one of the important risk factors for infective complications after RIRS [18]. The bacterial load contained in the stone and the risk of postoperative infective complications was reported to increase as the stone burden increases [19]. A retrospective study on 221 patients who underwent RIRS showed that the stone burden increased the risk of postoperative infective complications by prolonging the surgical duration and that the risk increased 11 -fold in cases with a surgical duration $>61$ min [20]. Alezra et al. [21] reported that the threshold value for this surgical duration was $70 \mathrm{~min}$. According to another study, a prolonged surgical duration was found to have a cumulative effect in increasing the pyelovenous reflux and transfer of bacteria from the stone to the systemic circulation, thereby constituting the main mechanism for postoperative infective complications [22]. According to our study, a surgical duration of $>60$ min increased the risk of infective complications 1.9 times and was found to be an independent risk factor. If the stone burden is $>200 \mathrm{~mm}^{2}$, more infective complications are seen. Despite this, the stone burden has not been determined to be an independent risk factor for infective complications. Accordingly, it can be concluded that the increase in the infective complication rate in cases with a high stone burden is due to the prolonged duration of the surgery.

In a study on 337 patients in whom risk factors for SIRS and fever occurring after RIRS were evaluated, the mean age of patients who developed SIRS was $51.2 \pm 10.5$ years, whereas it was found to be $53.1 \pm 13.3$ years in patients who did not; the difference was not statistically significant [19]. Similarly, in another prospective study, no difference was found between the mean age of patients with and without postoperative symptomatic urinary 
tract infection [23]. In our study, postoperative infective complications occurred 1.8 times frequently in patients aged $>50$ years compared with those aged $<50$ years, and this difference was statistically significant. Due to the higher number of patients in our study compared with that in other studies, we believe that achieving such a result may be related to the weakening of the immune system and increased susceptibility to clinical infections.

When the relationship between a history of treated urinary tract infection and postoperative infective complications was examined in a multicenter prospective study by Cai et al. [23] it was found that a history of treated urinary tract infection was not a risk factor. This is supported by the study of Li et al. [19]. However, Grabe et al. [24] reported that previous urinary tract infection is an important risk factor. In contrast, no such relationship was found in our study.

Congenital kidney anomalies have been shown to be risk factors for postoperative infective complications in some studies $[5,25]$. In our study, only fever was observed in 1 of the 14 patients with congenital kidney anomalies, whereas no infective complications were observed in others. However, the low number of patients with congenital kidney anomalies in our study makes it difficult to comment on this issue.

Some measures can be taken to prevent infective complications after RIRS. A recent review recommends preoperative urine culture, shortening the operation time, and applying RIRS in more than one session for large stones. Based on the results of the present study and a previous report [26], we recommend that the duration of the RIRS should not exceed $60 \mathrm{~min}$.

Specific scoring systems should be developed to predict infective complications after RIRS. However, according to the results of our study, the R.I.R.S score can be used to predict infective complications after RIRS. We recommend using this scoring system for risk assessment, especially in elderly patients and in cases where the operation is expected to take a long time.

Our study has some limitations. First, this is a retrospective study. Second, all surgeries were performed in a single center. In addition, although intrarenal pressure and irrigation fluid volume are important risk factors for infective complications, the lack of data regarding this information is a handicap in our study.

\section{Conclusion}

We conclude that the R.I.R.S. score can be used to predict infective complications after RIRS. However, considering its low selectivity, we believe that the development of new parameters or unique scoring systems is warranted in the future.

\section{Statement of Ethics}

This study protocol was approved by the local Ethics Committee (Approval No. E1-20-451).

\section{Conflict of Interest Statement}

The authors have no conflicts of interest to declare.

\section{Funding Sources}

None.

\section{Author Contributions}

S.S.: writing the manuscript. S.S., C.O., and Y.A.: protocol development and preliminary data analysis. Y.K., B.K.A., and C.S.G.: development of the protocol and analytical framework for the study and the revision of the manuscript.

\section{Data Availability Statement}

The data that support the findings of this study are available in the Figshare Repository at https://figshare.com/s/edc07fcb676536ec271e.

\section{References}

1 Chung DY, Kang DH, Cho KS, Jeong WS, Jung $\mathrm{HD}$, Kwon JK, et al. Comparison of stone-free rates following shock wave lithotripsy, percutaneous nephrolithotomy, and retrograde intrarenal surgery for treatment of renal stones: a systematic review and network meta-analysis. PLoS One. 2019;21(2):14.
2 Aboumarzouk OM, Monga M, Kata SG, Traxer O, Somani BK. Flexible ureteroscopy and laser lithotripsy for stones $>2 \mathrm{~cm}$ : a systematic review and meta -analysis. J Endouro. 2012;26(10):1257-63.

3 Lim SH, Jeong BC, Seo SI, Jeon SS, Han DH Treatment outcomes of retrograde intrarenal surgery for renal stones and predictive factors of stone-free. Korean J Urol. 2010;51(11):777-82. 
4 Jiang K, Sun F, Zhu J, Luo G, Zhang P, Ban Y, et al. Evaluation of three stone-scoring systems for predicting SFR and complications after percutaneous nephrolithotomy: a systematic review and meta-analysis. BMC Urol. 2019;119(1):57.

5 Resorlu B, Unsal A, Gulec H, Oztuna D. A new scoring system for predicting stone-free rateafter retrograde intrarenal surgery: the resorlu-unsal stone score. Urology. 2012; 80(3):512-8.

6 Jung JW, Lee BK, Park YH, Lee S, Jeong SJ, Lee SE, et al. Modified seoul national university renal stone complexity score for retrograde intrarenal surgery. Urolithiasis. 2014; 42(4):335-40.

7 Xiao Y, Li D, Chen L, Xu Y, Zhang D, Shao Y, et al. The R.I.R.S. scoring system: an innovative scoring system for predicting stone-free rate following retrograde intrarenal surgery. BMC Urol. 2017;17(1):105.

8 Uchida Y, Takazawa R, Kitayama S, Tsujii T. Predictive risk factors for systemic inflammatory response syndrome following ureteroscopic laser lithotripsy. Urolithiasis. 2018; 46(4):375-81.

9 Labadie K, Okhunov Z, Akhavein A, Moreira $\mathrm{DM}$, Moreno-Palacios J, Junco MD, et al. Evaluation and comparison of urolithiasis scoring systems in percutaneous kidney stone surgery. J Urol. 2015;193(1):154-9.

10 Okhunov Z, Friedlander JI, George AK, Duty $\mathrm{BD}$, Moreira DM, Srinivasan AK, et al. S.T.O.N.E. nephrolithometry: novel surgical classification system for kidney calculi. Urology. 2013;81(6):1154-9.

11 Smith A, Averch TD, Shahrour K, Opondo D, Daels FP, Labate G, et al. A nephrolithometric nomogram to predict treatment success of percutaneous nephrolithotomy. J Urol. 2013; 190(1):149-56.
12 Thomas K, Smith NC, Hegarty N, Glass JM. The Guy's stone score: grading the complexity of percutaneous nephrolithotomy procedures. Urology. 2011;78(2):277-81.

13 Türk C, Neisius A, Petřík A, Seitz C, Skolarikos A, Somani B, et al. EAU guidelines on urolithiasis. EAU guidelines; 2020.

14 Berardinelli F, De Francesco P, Marchioni M, Cera N, Proietti S, Hennessey D, et al. Infective complications after retrograde intrarenal surgery: a new standardized classification system. Int Urol Nephrol. 2016;48(11):1757-62.

15 Mitsuzuka K, Nakano O, Takahashi N, Satoh M. Identification of factors associated with postoperative febrile urinary tract infection after ureteroscopy for urinary stones. Urolithiasis. 2016;44(3):257-62.

16 Singer M, Deutschman CS, Seymour CW, Shankar-Hari M, Annane D, Bauer M, et al. The third international consensus definitions for sepsis and septic shock (Sepsis-3). JAMA. 2016;315(8):801-10.

17 Chugh S, Pietropaolo A, Montanari E, Sarica K, Somani BK. Predictors of urinary infections and urosepsis after ureteroscopy for stone disease: a systematic review from eau section of urolithiasis (EULIS). Curr Urol Rep. 2020;21(4):16.

18 Fan S, Gong B, Hao Z, Zhang L, Zhou J, Zhang $Y$, et al. Risk factors of infectious complications following flexible ureterorenoscope with a holmium laser: a retrospective study. Int J Clin Exp Med. 2015;8:1252-9.

19 Li T, Sun XZ, Lai DH, Li X, He YZ. Fever and systemic inflammatory response syndrome after retrograde intrarenal surgery: risk factors and predictive model. Kaohsiung J Med Sci. 2018;34:400-8.
20 Demir DO, Doluoglu OG, Yildiz Y, Bozkurt S, Ayyildiz A, Demirbas A. Risk factors for infectious complications in patients undergoing retrograde intrarenal surgery. J Coll Physicians Surg Pak. 2019;29(6):558-62.

21 Alezra E, Lasselin J, Forzini T, Francois T, Viart L, Saint F. Prognostic factors for severe infection after flexible ureterorenoscopy: clinical interest of urine culture the day before surgery? Prog Urol. 2016;26:65-71.

22 Kumar S, Bag S, Ganesamoni R, Mandal AK, Taneja N, Singh SK. Risk factors for urosepsis following percutaneous nephrolithotomy: role of 1 week of nitrofurantoin in reducing the risk of urosepsis. Urol Res. 2012;40:79-86.

23 Cai C, Cocci A, Coccarelli F, Ruggera L, Lanzafame P, Caciagli P, et al. Infectious complications after laser vaporization of urinary stones during retrograde intrarenal surgery are not associated with spreading of bacteria into irrigation fluid but with previous use of fluoroquinolones. Eur Urol Focus. 2019; 7(19):53-7.

24 Grabe M, Botto H, Cek M, Tenke P, Wagenlehner FME, Naber KG, et al. Preoperative assessment of the patient and risk factors for infectious complications and tentative classification of surgical field contamination of urological procedures. World J Urol. 2012; 30(1):39-50.

25 Atis G, Resorlu B, Gurbuz C, Arikan O, Ozyuvali $\mathrm{E}$, Unsal A, et al. Retrograde intrarenal surgery in patients with horseshoe kidneys. Urolithiasis. 2013;41:79-83.

26 Bhanot R, Pietropaolo A, Tokas T, Kallidonis P, Skolarikos A, Keller EX, et al. Predictors and strategies to avoid mortality following ureteroscopy for stone disease: a systematic review from European Association of Urologists Sections of Urolithiasis (EULIS) and Uro-technology (ESUT). Eur Urol Focus. 2021;S2405-4569(21):00057-2. 